\section{Acesso à assistência oncológica: mapeamento dos fluxos origem-destino das internações e dos atendimentos ambulatoriais. $O$ caso do câncer de mama}

\author{
Access to cancer care: mapping hospital \\ admissions and high-complexity outpatient \\ care flows. The case of breast cancer
}

Evangelina Xavier Gouveia de Oliveira ${ }^{1}$

Enirtes Caetano Prates Melo 2

Rejane Sobrino Pinheiro 3

Cláudio Pompeiano Noronha 4

Marilia Sá Carvalho 5

\title{
Introdução
}

1 Instituto Brasileiro de Geografia e Estatística, Rio de Janeiro, Brasil.

2 Escola de Enfermagem Alfredo Pinto, Universidade Federal do Estado do Rio de Janeiro, Rio de Janeiro, Brasil. 3 Instituto de Estudos em Saúde Coletiva, Universidade Federal do Rio de Janeiro, Rio de Janeiro, Brasil. 4 Instituto Nacional de Câncer, Rio de Janeiro, Brasil. 5 Escola Nacional de Saúde Pública Sergio Arouca, Fundação Oswaldo Cruz, Rio de Janeiro, Brasil.

Correspondência E. C. P. Melo Escola de Enfermagem Alfredo Pinto, Universidade Federal do Estado do Rio de Janeiro.

Rua Xavier Sigaud 290, sala $504 / 505$, Rio de Janeiro, $R J$ 22240-290, Brasil. enirtes@globo.com
This study analyzes the flow of patients with

\section{Abstract} breast cancer treated in Brazil's Unified National Health System (SUS) by type of treatment (surgery, radiotherapy, and chemotherapy). Hospital and outpatient services networks were identified based on data from the National Information System for Inpatient Care (SIH), and the National Information System for Outpatient Cancer Care, for 2005-2006, using TabWin and TerraView. Health services networks reach most of the country, and few municipalities are not connected to a network. However, treatment is highly concentrated in the largest cities, and even the latter show evidence of service shortages. Furthermore, a large proportion of patients live more than $150 \mathrm{~km}$ from the respective service. Network identification is important for planning and improving services distribution, since geographic access is a relevant issue for treatment outcome. Reduction of morbidity and mortality requires early identification, and appropriate and prompt treatment can reduce the impacts of the disease.

Breast Neoplasms; Spatial Analysis; Health Services Accessibility; Informations Systems
O câncer de mama é o câncer mais frequente entre as mulheres em todo o mundo, com uma estimativa de ocorrência de aproximadamente 1,3 milhão de novos casos com lesão invasiva diagnosticados em 2007. Com uma estimativa de 465 mil mortes para o mesmo período 1 , representa 0 câncer com o maior volume de mortes em mulheres em todo o mundo.

No Brasil também é o câncer mais incidente com uma ocorrência de aproximadamente 50 mil casos novos a cada ano, ou $28 \%$ do total das neoplasias malignas femininas, excluindo os tumores de pele não melanoma. Este padrão de ocorrência apresenta variações regionais importantes no país, com taxas de incidência mais elevadas nas regiões Sul e Sudeste, ao mesmo tempo em que as menores incidências são verificadas na Região Norte. O câncer de mama também é o líder na mortalidade feminina por câncer com 11 mil mortes registradas no país em 2007. O crescimento desta mortalidade é observado a partir do final da década de setenta, quando o câncer de mama assume a posição de principal causa de morte por câncer em mulheres, no Brasil (15,5\%), acompanhando o padrão mundial 2. A mortalidade é maior, principalmente, em regiões onde a incidência é elevada e os recursos médicos para um diagnóstico precoce e tratamento adequado são de difícil acesso ou mesmo inexistentes. $\mathrm{O}$ desempenho dos programas de rastreamento e 
o sub-registro de casos também exercem forte influência sobre o perfil de morbimortalidade da doença no Brasil e em outras partes do mundo 3.

Embora as neoplasias malignas sejam a segunda causa de morte no país, as estratégias para seu controle enfrentam problemas que afetam desde os mecanismos de formulação de políticas, até a mobilização da sociedade, incluindo a organização e o desenvolvimento das ações e serviços e as atividades de ensino e pesquisa. Dois aspectos caracterizam o câncer como um problema de saúde pública no Brasil. Primeiro, o aumento gradativo da incidência e mortalidade por câncer, proporcionalmente ao crescimento demográfico, o envelhecimento populacional e ao desenvolvimento socioeconômico. Segundo, o desafio que isso representa para o sistema de saúde no sentido de garantir-se o acesso pleno e equilibrado da população ao diagnóstico e tratamento dessa doença.

A utilização dos serviços de saúde está ligada a características da oferta e à conduta das pessoas frente à morbidade e aos serviços. O padrão de utilização pode variar segundo sexo, grupos etários, grupos sociais, problemas de saúde, procedimentos específicos e áreas geográficas. Barry \& Breen ${ }^{4}$ observaram que, para portadoras de câncer de mama, um dos fatores determinantes de doença avançada quando do diagnóstico, além dos fatores sócio-demográficos, foi a residência em área mal servida por serviços de saúde.

Um adequado planejamento e avaliação do setor saúde terão de levar em conta a identificação dos pólos de atração, a regionalização do atendimento, as distâncias percorridas pela população na busca pela assistência, e os volumes envolvidos nestes deslocamentos. A análise de redes é uma forma de investigar a questão das relações entre local de residência e local do serviço de saúde. A configuração das ligações revela a estrutura da rede 5 . Redes em árvore caracterizam-se pela limitação das conexões entre seus diversos ramos, ao passo que redes organizadas em malha - em que cada nó se liga a vários outros - permitem percorrer caminhos variados entre os pontos, de modo que os diversos ramos da rede estão interconectados 6 . Nesse sentido, observe-se que padrões de ligações hierárquicas, com poucas ligações em malha, são reconhecidos como eficiente mecanismo de racionamento de serviços escassos 7 .

A Política Nacional de Atenção Oncológica: Promoção, Prevenção, Diagnóstico, Tratamento, Reabilitação e Cuidados Paliativos, instituída em 2005 (Portaria no ${ }^{\circ}$. 2.439/GM de 8 de dezembro de 2005) ${ }^{8}$, destaca a "necessidade de se estruturar uma rede de serviços regionalizada e hierarquizada que garanta atenção integral à população, bem como o acesso a consultas e exames para o diagnóstico do câncer". Embora o período aqui estudado seja anterior à implantação desta política, vale notar que a Portaria $n^{\circ} 3.535 / G M 9$, de 2 de setembro de 1998, já definia critérios para cadastramento de Centros de Alta Complexidade em Oncologia, atribuindo às secretarias de saúde (dos estados, do Distrito Federal e dos municípios) o estabelecimento dos fluxos e referências para o atendimento a pacientes com câncer.

Assim, o principal objetivo deste estudo é analisar o fluxo de pacientes com câncer de mama, atendidas no âmbito do SUS, em todo o país, segundo o tipo de tratamento recebido (cirurgia, radioterapia e quimioterapia), utilizando Sistema de Informações Geográficas e metodologia de redes.

\section{Método}

Este é um estudo ecológico que analisa as cirurgias e os atendimentos ambulatoriais, em hospitais públicos e contratados pelo SUS, sendo a população do estudo constituída pelas mulheres com diagnóstico principal de câncer de mama (C50 e D05 da 10a revisão da Classificação Internacional de Doenças - CID-10) atendidas na rede de saúde vinculada ao SUS.

Foram utilizadas as bases de dados, disponibilizadas pelo Instituto Nacional de Câncer e pelo Departamento de Informática do SUS (DATASUS; http://www.datasus.gov.br), referentes ao SubSistema de Informações Hospitalares (SIH), bem como os dados do Sistema de Informações Ambulatoriais do SUS (SIA-SUS), módulo de alta complexidade para atendimento oncológico (APAC/ONCO), em todo Brasil.

O processo de seleção de cirurgias e de procedimentos elegíveis em cada uma das bases foi realizado a partir do código da CID-10. Estudaramse as cirurgias, realizadas de janeiro a dezembro de 2005, segundo o município de tratamento e o de residência dos pacientes, e os atendimentos ambulatoriais (quimioterapia ou radioterapia) relativos ao período de julho de 2005 a junho de 2006. Foram selecionadas como cirurgia de mama aquelas que caracterizam procedimentos do tratamento inicial para mama: mastectomia radical com linfadenectomia, mastectomia simples, ressecção de lesão de mama, setorectomia, setorectomia com esvaziamento ganglionar, linfadenectomia radical axilar unilateral e bilateral, biópsia de mama.

Um par origem-destino, entre local de residência e de atendimento, define uma ligação e o número de pessoas que se deslocam configura um fluxo. Para a identificação de redes de aten- 
ção em oncologia, utilizou-se o método do fluxo dominante que, para cada município, é o maior fluxo de saída 7,10. A classificação das ligações, segundo a tipologia dos fluxos baseada naquela proposta por Rabino \& Occelli 11, avalia o grau de interconexão das redes. Com isto, além do fluxo dominante, os demais são também considerados, evitando-se perda de informação e permitindo examinar os relacionamentos internos e externos de cada rede.

A tipologia aqui adotada classifica os fluxos em diferentes tipos, segundo acompanhem, ou não, a estrutura da rede. Ao identificar uma rede pelo método do fluxo dominante, estabelece-se uma hierarquia entre os centros (nós) da rede, que são classificados em níveis. Fluxos hierárqui- cos são os que acompanham a estrutura da rede definida pelo fluxo dominante, e fluxos transversais são os que ocorrem entre diferentes redes, ou entre diferentes ramos de uma mesma rede. Os fluxos que ligam centros de mesmo nível são horizontais, os que se dirigem para centros de nível superior são ascendentes, os que, ao contrário, demandam um centro de nível mais baixo, são descendentes. Além disso, em termos da estrutura da rede, é possível verificar se os fluxos ascendentes ou descendentes estabelecem ligação direta com o próximo nível hierárquico, ou se saltam um ou mais níveis, sendo então chamados de fluxos em "curto circuito" 12 (Figura 1).

A unidade geográfica de análise foi o município. O processamento dos dados e mapeamento

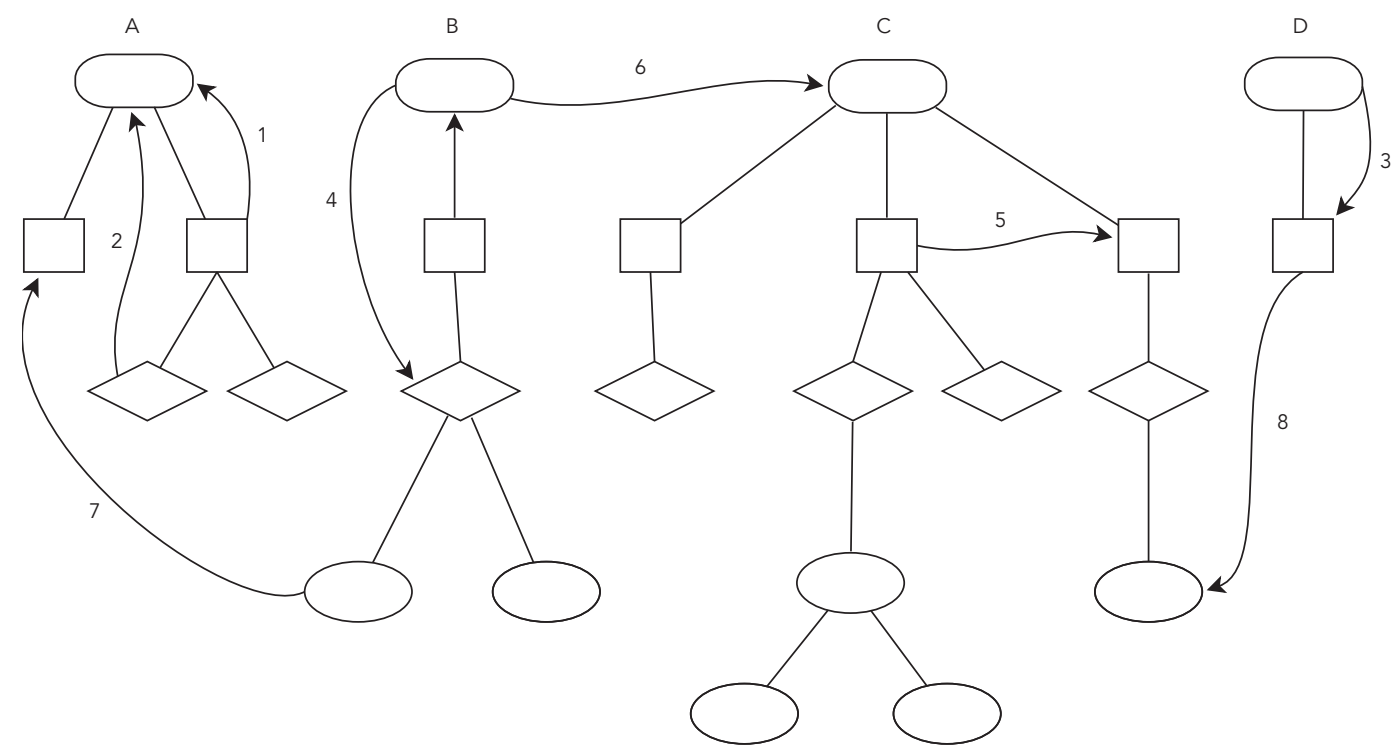

Redes A, B, C, D

Classificação dos fluxos:

1. Hierárquico-ascendente direto

2. Hierárquico-ascendente em curto circuito

3. Hierárquico-descendente direto

4. Hierárquico-descendente em curto circuito

5. Transversal horizontal mesma rede

6. Transversal horizontal entre redes

7. Transversal ascendente entre redes

8. Transversal descendente entre redes

* Adaptado de Rabino \& Occelli 11. 
dos resultados foi feito com os programas de domínio público R (The R Foundation for Statistical Computing, Viena, Áustria; http: / /www.r-project. org), TabWin (DATASUS. http://portal.saude.gov. $\mathrm{br} /$ portal/se/datasus/area.cfm?id_area=732) e TerraView (Instituto Nacional de Pesquisas Espaciais; http://www.dpi.inpe.br/terraview). O TabWin tem opção para tabular as informações segundo o local de residência e o do atendimento, viabilizando a análise dos fluxos segundo origem e destino 13. No TerraView, usou-se o plug-in de fluxos, com a opção de integrar um município a uma rede se o seu maior fluxo representa pelo menos $5 \%$ do total de pacientes ali residentes.

O projeto foi submetido e aprovado pelo Comitê de Ética em Pesquisa da Escola Nacional de Saúde Pública Sergio Arouca, Fundação Oswaldo Cruz.

\section{Resultados}

No Brasil foram realizadas no SUS, durante o período estudado, 20.828 cirurgias, 37.000 internações, 831.759 procedimentos ambulatoriais de quimioterapia e 43.939 de radioterapia em mulheres com diagnóstico de câncer de mama. Quase $40 \%$ do volume total de atendimentos estiveram concentrados em apenas sete capitais - São Paulo, Rio de Janeiro, Belo Horizonte (Minas Gerais), Porto Alegre (Rio Grande do Sul), Curitiba (Paraná), Salvador (Bahia) e Fortaleza (Ceará) -, que constituem os grandes centros de tratamento da doença, atendendo, majoritariamente, seus residentes (Tabela 1 ).

No Brasil, mais da metade do atendimento foi local, isto é, realizado no próprio município de residência: $56,5 \%$ das cirurgias, $54,6 \%$ dos atendimentos de quimioterapia e $48,7 \%$ dos atendimentos de radioterapia não configuraram deslocamento.

\section{Cirurgias}

Na investigação da rede de atenção hospitalar para o câncer de mama, verificou-se que 529 municípios internaram pessoas nesta especialidade, residentes em 2.816 municípios (50,6\% do total), correspondendo a 20.828 cirurgias no ano de 2005. Algumas capitais mostraram um perfil de pólo de atenção um pouco mais acentuado, com mais de $40 \%$ do atendimento de não-residentes, caso de Recife (Pernambuco), Porto Alegre, Belo Horizonte e Curitiba, com 71,4\%, 60\%, 53,7\% e $50,7 \%$ de não-residentes atendidos, respectivamente. Dos 3.714 pares de origem-destino, cerca de $11,7 \%$ (Tabela 2) representam internações no próprio município de residência, o que corresponde a $55,8 \%$ do total de cirurgias. Das cirurgias locais, 50\% são realizadas em apenas 12 municípios [São Paulo, Rio de Janeiro, Salvador, Brasília, Fortaleza, Porto Alegre, Belo Horizonte, Recife, Manaus (Amazonas), Curitiba, Maceió (Alagoas) e Belém (Pará)], e metade deste valor (25,4\%) está concentrada no Rio de Janeiro e em São Paulo. A mediana das distâncias percorridas é de 67 km, e $75 \%$ dos deslocamentos entre os pares têm distância inferior a $151 \mathrm{~km}$, correspondendo a $91,7 \%$ das cirurgias. Apenas $2,4 \%$ da população brasileira moram nos 301 municípios (5,4\% dos municípios brasileiros) situados a mais de $150 \mathrm{~km}$ de distância de um centro onde foi possível realizar a cirurgia, e para residentes em 242 deles, não houve registro de atendimento no período.

Distribuição percentual da assistência a pacientes de câncer de mama, segundo tipo de tratamento e local de atendimento, capitais selecionadas e Brasil, 2005-2006.

\begin{tabular}{|c|c|c|c|c|c|c|c|c|c|}
\hline \multirow[t]{3}{*}{ Capitais (Estado) } & \multicolumn{3}{|c|}{ Cirurgia } & \multicolumn{3}{|c|}{ Quimioterapia } & \multicolumn{3}{|c|}{ Radioterapia } \\
\hline & \multicolumn{2}{|c|}{ Fluxo } & \multirow[t]{2}{*}{ Brasil } & \multicolumn{2}{|c|}{ Fluxo } & \multirow[t]{2}{*}{ Brasil } & \multicolumn{2}{|c|}{ Fluxo } & \multirow[t]{2}{*}{ Brasil } \\
\hline & Local & Externo & & Local & Externo & & Local & Externo & \\
\hline São Paulo (São Paulo) & 79,0 & 21,0 & 12,0 & 79,1 & 20,9 & 9,8 & 82,9 & 17,1 & 9,4 \\
\hline Rio de Janeiro (Rio de Janeiro) & 65,8 & 34,2 & 7,4 & 64,8 & 35,2 & 6,4 & 70,9 & 29,1 & 5,5 \\
\hline Porto Alegre (Rio Grande do Sul) & 40,0 & 60,0 & 4,1 & 44,4 & 55,6 & 4,8 & 32,1 & 67,9 & 4,8 \\
\hline Salvador (Bahia) & 61,0 & 39,0 & 4,4 & 63,9 & 36,1 & 3,7 & 60,2 & 39,8 & 3,9 \\
\hline Belo Horizonte (Minas Gerais) & 46,3 & 53,7 & 3,0 & 48,9 & 51,1 & 6,0 & 40,2 & 59,8 & 4,7 \\
\hline Fortaleza (Ceará) & 72,4 & 27,6 & 3,3 & 72,5 & 27,5 & 4,8 & 64,7 & 35,3 & 3,9 \\
\hline Curitiba (Paraná) & 49,3 & 50,7 & 1,7 & 51,4 & 48,6 & 2,9 & 53,3 & 46,7 & 3,1 \\
\hline Recife (Pernambuco) & 28,6 & 71,4 & 4,1 & 36,1 & 63,9 & 2,8 & 30,2 & 69,8 & 3,1 \\
\hline Brasil & 56,5 & 43,5 & 100,0 & 54,6 & 45,4 & 100,0 & 48,7 & 51,3 & 100,0 \\
\hline
\end{tabular}


Distribuição das ligações segundo classificação para atendimento ao câncer de mama no Brasil, 2005-2006.

\begin{tabular}{|c|c|c|c|c|c|c|c|c|c|}
\hline \multirow[t]{2}{*}{ Tipo de ligação } & \multicolumn{3}{|c|}{ Cirurgia } & \multicolumn{3}{|c|}{ Quimioterapia } & \multicolumn{3}{|c|}{ Radioterapia } \\
\hline & $\begin{array}{c}\text { Ligações } \\
(\%)\end{array}$ & $\begin{array}{c}\text { Fluxos } \\
\text { (\%) }\end{array}$ & $\begin{array}{l}\text { Distância } \\
\text { média }(\mathrm{km})\end{array}$ & $\begin{array}{c}\text { Ligações } \\
\text { (\%) }\end{array}$ & $\begin{array}{c}\text { Fluxos } \\
\text { (\%) }\end{array}$ & $\begin{array}{c}\text { Distância } \\
\text { média (km) }\end{array}$ & $\begin{array}{c}\text { Ligações } \\
\text { (\%) }\end{array}$ & $\begin{array}{c}\text { Fluxos } \\
(\%)\end{array}$ & $\begin{array}{c}\text { Distância } \\
\text { média }(\mathrm{km})\end{array}$ \\
\hline Ligação local & 11,7 & 55,8 & 0 & 1,8 & 54,6 & 0 & 2,6 & 48,7 & 0 \\
\hline Hierárquico ascendente direta & 72,9 & 39,9 & 139 & 63,1 & 38,9 & 155 & 79,8 & 44,5 & 149 \\
\hline Hierárquico ascendente em curto-circuito & 0,8 & 0,3 & 161 & 2,1 & 0,4 & 242 & 2,1 & 0,8 & 169 \\
\hline Hierárquico descendente direta & 0,4 & 0,3 & 19 & 0,0 & 0,2 & 32 & 0,1 & 0,3 & 24 \\
\hline Hierárquico descendente em curto-circuito & - & - & - & 0,3 & 0,0 & 26 & - & - & - \\
\hline Transversal horizontal mesma rede & 4,2 & 1,3 & 51 & 5,1 & 1,3 & 86 & 1,9 & 0,6 & 91 \\
\hline Transversal horizontal entre redes & 2,1 & 0,6 & 201 & 3,8 & 0,8 & 275 & 2,6 & 1,6 & 281 \\
\hline Transversal ascendente entre redes & 7,7 & 1,8 & 198 & 23,6 & 3,5 & 283 & 10,6 & 2,6 & 219 \\
\hline Transversal descendente entre redes & 0,1 & 0,0 & 49 & 0,2 & 0,2 & 93 & 0,3 & 0,9 & 144 \\
\hline Total & 100,0 & 100,0 & 123 & 100,0 & 100,0 & 185 & 100,0 & 100,0 & 155 \\
\hline
\end{tabular}

As cirurgias de câncer de mama conformam 110 redes. Em termos de volume de internações, as redes de São Paulo e do Rio de Janeiro são responsáveis por $19,4 \%$ do total. Juntando-se as demais redes que realizaram mais de 500 cirurgias no ano [as de Salvador, Porto Alegre, Recife, Fortaleza, Belo Horizonte e Barretos (São Paulo)], esse valor atinge $40,9 \%$ do total. Seis redes destacam-se por abranger mais de 100 municípios, as de Barretos, Salvador, Recife, Belo Horizonte, Porto Alegre e Teresina (Piauí) que, reunindo 832 municípios, realizaram 19,3\% das cirurgias. Nas redes, de modo geral, o centro principal tende a concentrar a maior parte do atendimento. No conjunto das maiores redes, uma participação mais expressiva de subcentros foi observada apenas nas redes de Campinas (São Paulo) e de Curitiba, cuja proporção de cirurgias na cidade central foi de $52,4 \%$ e $67,4 \%$, respectivamente.

Por construção, o padrão das redes é dado pelas ligações hierárquicas. Entre as ligações transversais, predominam as ascendentes entre as redes, que demandam, principalmente, os centros das maiores redes (Figura 2 e Tabela 2).

\section{Ambulatoriais}

Foram analisados 831.759 procedimentos de quimioterapia aplicados a pacientes residentes em 4.572 municípios brasileiros, e 43.939 procedimentos de radioterapia, de pacientes residentes em 2.840 municípios. Do total, 131 municípios realizam procedimentos de quimioterapia e 89 de radioterapia.

Dos 7.123 pares de origem-destino que se referem ao atendimento para quimioterapia, ape- nas $1,8 \%$ (Tabela 2) correspondem a tratamento de residentes no município de localização do serviço, mas esta parcela representa $54,6 \%$ do total de atendimentos realizados. Dos atendimentos locais, $53 \%$ são realizadas em apenas 10 municípios [São Paulo, Rio de Janeiro, Fortaleza, Belo Horizonte, Salvador, Porto Alegre, Curitiba, Natal (Rio Grande do Norte), Recife e Goiânia (Goiás)], e pouco menos da metade deste valor $(43,6 \%)$ está concentrada no Rio de Janeiro e em São Paulo. A mediana das distâncias percorridas é de $108 \mathrm{~km}$, e $90,6 \%$ dos atendimentos foram prestados a residentes dentro de um raio de $150 \mathrm{~km}$ do serviço. Nos 1.315 municípios (23,6\% dos municípios brasileiros) situados a mais de $150 \mathrm{~km}$ de distância de um centro onde a quimioterapia estava disponível, moram $12,9 \%$ da população brasileira e para 443 deles não houve registro de atendimento no período.

Em relação à radioterapia, dos 3.462 pares de origem-destino, $2,6 \%$ correspondem a tratamento de residentes no município de localização do serviço, e esta parcela representa $48,7 \%$ do total de atendimentos realizados. Dos atendimentos locais, 50,8\% são realizadas em apenas 9 municípios (São Paulo, Rio de Janeiro, Fortaleza, Salvador, Belém, Belo Horizonte, Curitiba, Porto Alegre e Goiânia), e pouco menos da metade deste valor $(47,5 \%)$ está concentrada no Rio de Janeiro e em São Paulo. A mediana das distâncias percorridas é de $94 \mathrm{~km}$, e $88,8 \%$ dos atendimentos foram prestados a residentes dentro de um raio de $150 \mathrm{~km}$ do serviço. Da mesma forma que no caso anterior, 15,5\% da população brasileira moram nos 1.530 municípios $(27,5 \%$ dos municípios brasileiros) situados a mais de $150 \mathrm{~km}$ de distância de 
um centro onde foi possível realizar o tratamento e, para residentes em 981 destes, não houve registro de atendimento no período.

Os procedimentos de quimioterapia para o câncer de mama conformam 88 redes. Dez delas são responsáveis por $50,8 \%$ do atendimento, as de São Paulo, Rio de Janeiro, Belo Horizonte, Porto Alegre, Fortaleza, Salvador, Curitiba, Recife, Campinas e Barretos. A concentração do atendimento nas redes de quimioterapia de São Paulo e do Rio de Janeiro é menor do que no caso da cirurgia, correspondendo a 17,6\%. Quatorze redes abrangem mais de 100 municípios, as de Teresina, Belo Horizonte, Salvador, Barretos, Goiânia, Fortaleza, Recife, João Pessoa (Paraíba), Porto Alegre, Natal, Florianópolis (Santa Catarina), Curitiba, Cascavel (Paraná) e Jaú (São Paulo). Estas redes reúnem 2.405 municípios, e realizaram 43,6\% dos atendimentos. Neste conjunto, uma expressiva participação de subcentros foi observada apenas nas redes de São Paulo, Rio de Janeiro, Porto Alegre, Curitiba e Jaú, cujas proporções de quimioterapia na cidade central foram de $27,6 \%$, $41,5 \%, 42,9 \%, 46,8 \%$ e $49,8 \%$, respectivamente.

A radioterapia conformou 70 redes, sendo dez delas, as de São Paulo, Rio de Janeiro, Porto Alegre, Belo Horizonte, Salvador, Fortaleza, Barretos, Curitiba, Recife e Botucatu (São Paulo), responsáveis por cerca de $50 \%$ do atendimento (as duas primeiras com 18,3\%). Apenas as redes de Barretos, Salvador, Belo Horizonte, Teresina, Goiânia, Porto Alegre e Recife abrangem mais de 100 municípios (934, no conjunto) realizando $25,1 \%$ dos atendimentos. Entre as grandes redes, apenas a de Botucatu e São Paulo têm maior participação de subcentros, com 48,9\% e 50,7\% de tratamentos realizados no principal centro, respectivamente.

Em relação à classificação dos fluxos de pacientes nas redes de quimioterapia e de radioterapia, além da predominância das ligações hierárquicas, destacam-se secundariamente as ligações ascendentes entre redes. No caso da quimioterapia, em especial, com grande predominância de ligações ascendentes entre redes, aponta para ligações entre os municípios configurando um menor nível de organização da rede. Observa-se, além disto, que os deslocamentos para radioterapia são, em geral, menos extensos do que aqueles para quimioterapia (Figura 2, Tabela 2).

\section{Discussão}

Com o presente estudo, observou-se que, embora a rede para atendimento ao câncer de mama alcance a maior parte do território nacional, há vazios sanitários, sobretudo no Norte do país. Vale destacar que o método empregado não dimensiona a oferta ou a demanda e limita-se a investigar as ligações definidas pela presença do serviço, pelo que não avalia taxas de atendimento ou necessidade. Ainda assim, há forte indicação de escassez de oferta de atendimento na maior parte do país, até mesmo nas regiões onde a oferta de serviços é maior do que na maioria dos municípios. Com relação a vazios de atendimento, observou-se que aproximadamente metade dos municípios não encaminhou qualquer paciente para a cirurgia de mama na rede pública e conveniada ao SUS, 17,8\% não encaminharam para a quimioterapia e $49 \%$ para a radioterapia. Ainda que estas áreas tendam a ser menos densamente povoadas, as dificuldades de acesso impõem barreiras que comprometem a qualidade da assistência às pessoas ali residentes, em especial no que se refere à prestação de tratamento adequado em tempo oportuno. Mesmo quando o tratamento está disponível, há evidências de sua concentração espacial: metade do volume total de atendimentos no país se deu em algumas capitais, em especial no Rio de Janeiro e em São Paulo, que constituem os grandes centros de atendimento para o tratamento da doença, responsáveis por cerca de um quinto do atendimento nacional, na sua maioria para seus residentes.

O maior percentual de municípios com mulheres recebendo quimioterapia em relação à cirurgia $(82,2 \%$ vs. 50,6\%) pode apontar para estadiamentos avançados de câncer de mama em que as pacientes não têm mais indicação de cirurgia, restando apenas o tratamento paliativo. Outra hipótese que pode ser levantada é que muitas mulheres em estadiamento inicial podem recorrer à realização de procedimento cirúrgico para retirada de tumor usando financiamento próprio, para agilidade no início do tratamento, cujos custos são bem menores que os do tratamento ambulatorial. Na seqüência do tratamento, com os maiores custos do tratamento ambulatorial, há necessidade de a paciente ter acesso à rede pública.

Observou-se, ainda, percentual elevado das mulheres cujo tratamento foi financiado pelo SUS residindo a mais de $150 \mathrm{~km}$ do local de atendimento. Sabendo que o tratamento é baseado em procedimentos freqüentes, os resultados sugerem que grande parcela das mulheres que obtiveram atendimento enfrentou dificuldades adicionais à própria doença, em função do extenso deslocamento que necessitaram fazer.

A prevenção e a identificação precoce são requisitos importantes para a redução das taxas de morbidade e mortalidade por câncer de mama. No entanto, uma vezidentificado o caso, o tratamento 
Figura 2

Redes de cirurgia, quimioterapia e radioterapia para câncer de mama e fluxos transversais.

2a) Cirurgias
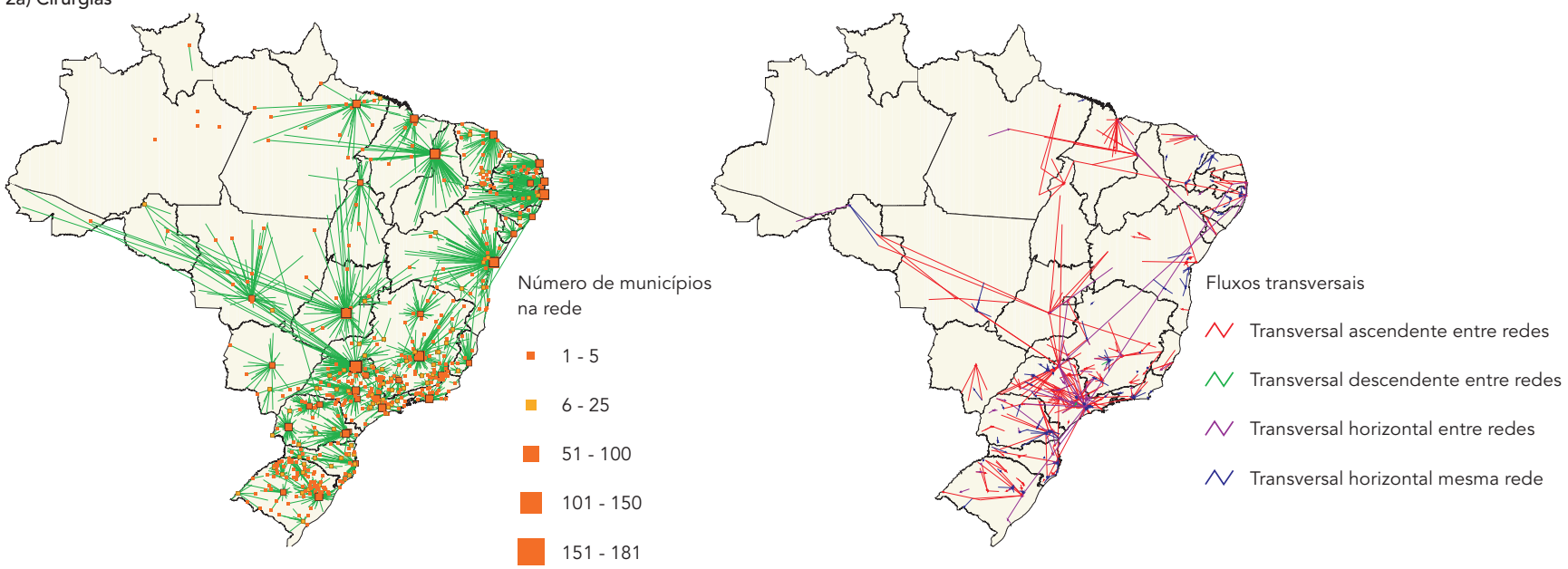

2b) Quimioterapia
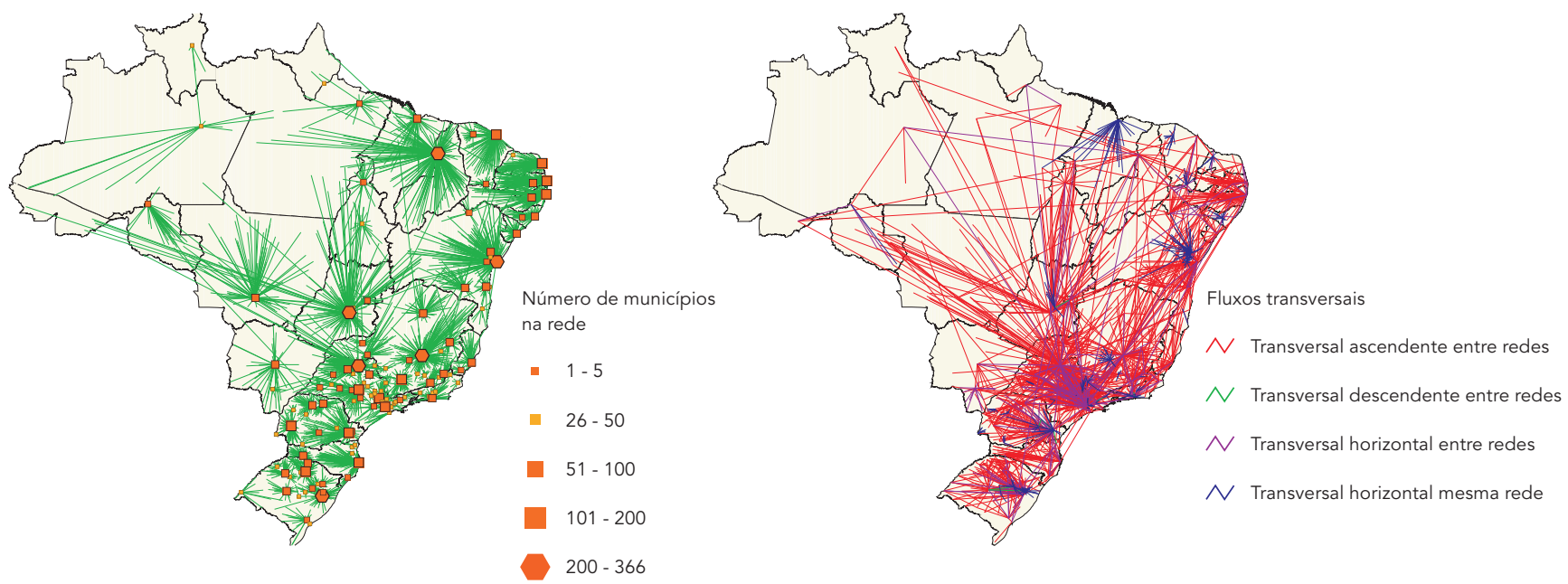

2c) Radioterapia
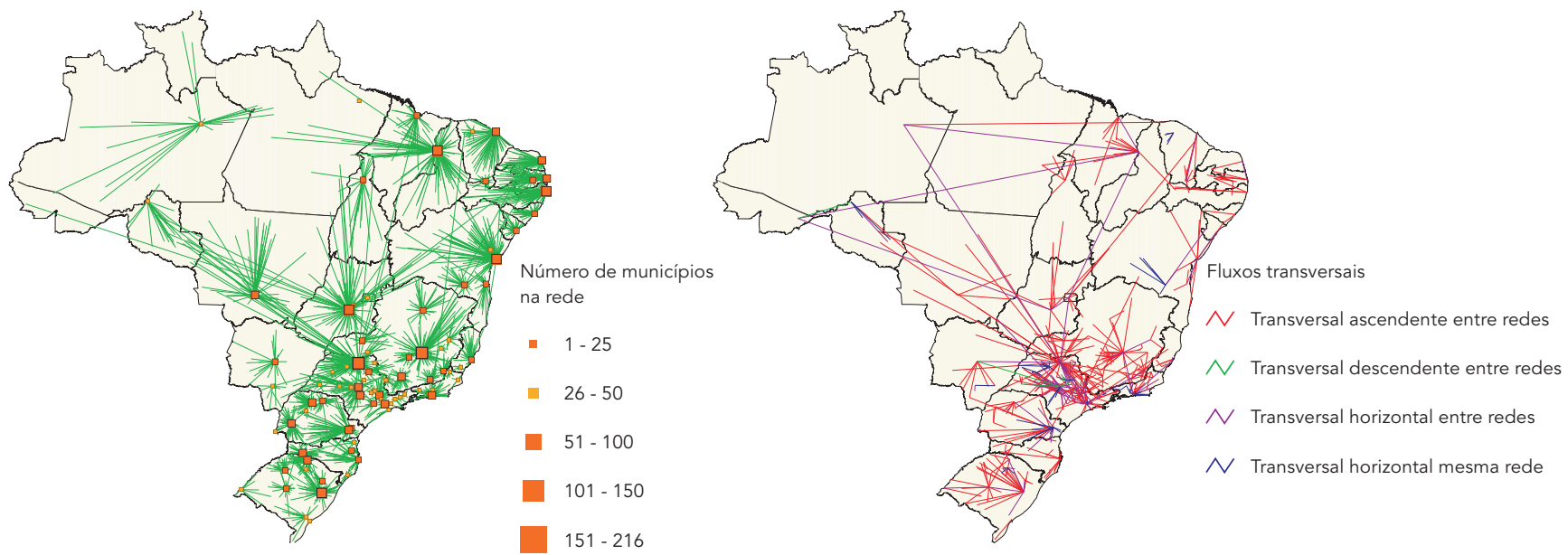
adequado e ágil concorrerá para a minimização dos impactos indesejados da doença 14,15. Nesse sentido, o acesso geográfico ao tratamento é de fundamental importância. O tratamento visa à cura, exceto nos casos de estadiamentos mais avançados, quando é indicado tratamento paliativo. A cirurgia para retirada do tumor é a primeira etapa do tratamento nos estadiamentos menores, seguida pelo tratamento ambulatorial, que é composto na maioria das vezes por séries de aplicações de quimioterapia ou radioterapia, levando à necessidade de organizar-se uma rede de serviços, com referências bem estabelecidas e logística definida para o tratamento completo 14 . Nos estadiamentos avançados, a redução do tumor para cirurgia (quimioterapia e/ou radioterapia neoadjuvante) ou mesmo para cuidado paliativo é amplamente indicada, apontando para a relevância do tratamento ambulatorial acessível em quantidade, tempo e localização às mulheres que dele necessitam. Uma vez que as unidades de saúde mostraram exercer uma atratividade em relação à população de sua cercania, o local de residência é uma informação relevante para identificação da população de referência e na organização da oferta de serviços de alta complexidade em câncer. Nessa perspectiva, estratégias para transporte e hospedagem dos pacientes têm papel destacado e devem ser consideradas.

Os resultados aqui apresentados evidenciam que o padrão de concentração do atendimento ambulatorial ao câncer de mama é menos acentuado do que aquele observado no tratamento cirúrgico. A configuração das redes de atenção representa inúmeras possibilidades de conexão que podem favorecer, ou não, o acesso da população à assistência. A marcada limitação das ligações dos municípios àqueles que lhes são superiores na rede, em um quadro de escassez, pode representar, em vez de uma eficiente organização da rede, uma forma de racionamento que não admite alternativas. As ligações transversais indicam alternativas de atendimento, e podem apontar centros com potencial de expansão. Os três padrões encontrados apóiam a idéia de diferentes situações do tratamento, definidas a partir do estadiamento clínico e do tipo histopatológico. Em relação à cirurgia verifica-se uma menor participação de fluxos transversais. Em que pese a distribuição territorial de infraestrutura, acredita-se que nessa modalidade terapêutica haja uma maior participação do setor de saúde suplementar ou utilização de recursos próprios para a realização do procedimento cirúrgico.

No caso da radioterapia, a baixa participação de fluxos transversais guarda relação com a concentração do atendimento em poucos centros. Tal padrão parece condicionado pela complexa infra-estrutura necessária (equipamentos e instalações) para o funcionamento de serviços radioterápicos. E finalmente, para a quimioterapia há uma expressiva presença de fluxos transversais que indicam uma articulação menos hierarquizada e mais aberta da rede.

A diferença no padrão de ligações transversais, com a quimioterapia apresentando maior número de conexões entre centros de mesmo nível em uma mesma rede, é causada pela presença de redes agregando maior número de municípios, e mais extenso território, quando comparada à relativa fragmentação da rede de radioterapia. No conjunto, os resultados apontam a existência de sérios problemas de acesso da população aos serviços aqui considerados. Se, de um lado, a presença de fluxos transversais revela fragilidades na estruturação da rede assistencial que comprometem o planejamento e a regionalização da assistência, por outro, aponta interconexões entre os vários ramos das redes, complementando a rede hierárquica e facilitando o acesso. No caso da atenção oncológica a precocidade do diagnóstico e o tratamento adequado têm papel fundamental na sobrevida do paciente.

Pacientes com câncer necessitam superar barreiras sociais, econômicas e psicológicas. Assim como a proximidade do serviço de saúde interfere na precocidade do diagnóstico 16, a distância percorrida pelo paciente deve ser considerada no tratamento, já que este exige repetidas visitas aos serviços de saúde para atendimento ambulatorial e internação. Neste sentido, a localização do serviço e dos usuários, os meios de transporte disponíveis, a distância, o tempo e os custos envolvidos no deslocamento devem ser considerados na avaliação do padrão de acessibilidade 17. Em um país com as dimensões do Brasil, a distribuição desigual da população e dos serviços de saúde no território marca um desafio a ser enfrentado. Na atenção terciária, a concentração dos recursos diagnósticos e terapêuticos, desejável para garantir maiores volumes e melhor qualidade do atendimento, muitas vezes impõe extensos deslocamentos a uma parcela considerável da população. Para evitar a pulverização de esforços e de investimento, a rede pública e conveniada ao SUS prevê a realização de tratamento fora do domicílio (TFD), custeando passagens e diárias para paciente e acompanhante. É vedado o pagamento deste benefício para procedimentos incluídos no Piso da Atenção Básica, deslocamentos menores do que $50 \mathrm{~km}$ de distância ou aqueles no interior de regiões metropolitanas. Tal política confere maior grau de ajuste entre as necessidades dos usuários e a oferta de serviços de saúde, ainda que representem um recurso limitado. 
Variações regionais podem refletir, além dos atributos do lugar, padrões de desigualdade relacionados a características pessoais (sexo, idade, raça/etnia) e socioeconômicas (renda, escolaridade) dos indivíduos 18, que limitam os resultados alcançáveis por políticas igualitárias. Estudo americano realizado no Estado de Ohio verificou uma redução menor da mortalidade por câncer de mama entre mulheres idosas e negras 19 . Mesmo em sociedades mais homogêneas, como é o caso da Suécia, observou-se a influência de fatores socioeconômicos na sobrevida do câncer de mama, com marcadas diferenças entre mulheres com menos de 50 anos no momento do diagnóstico 20. A disparidade no acesso aos cuidados de saúde (levando a um estadiamento mais avançado no diagnóstico) é apontada por estudos anteriores como o principal fator relacionado às diferenças na tendência geral de declínio da mortalidade por câncer de mama entre os subgrupos populacionais ao longo da década de noventa 19,20,21.

A investigação de áreas de abrangência das unidades do SUS pela identificação das redes estabelecidas por sua efetiva utilização, e as indicações de possíveis regionalizações alternativas dadas pelos fluxos transversais, constituem ferramentas com aplicação potencialmente importante no planejamento e melhoria da distribuição dos serviços de acordo com as necessidades da população usuária.

\section{Resumo}

Este estudo analisa ofluxo de pacientes atendidas com câncer de mama, no Brasil, no âmbito do SUS, segundo o tipo de tratamento recebido. Foram identificadas redes de atenção oncológica com base nas informações do Sistema de Informações Hospitalares e do Sistema Informações Ambulatoriais de Alta Complexidade em Oncologia, relativas ao período 2005-2006, utilizando os programas TabWin e TerraView. O atendimento está amplamente distribuído pelo território nacional, com forte concentração nos maiores centros, $e$ indícios de escassez de atendimento mesmo nas regiões onde a oferta de serviços é maior. Grande proporção das pacientes reside a mais de $150 \mathrm{~km}$ do local de atendimento. A identificação das redes constitui ferramenta com aplicação importante no planejamento e na melhoria da distribuição dos serviços, considerando que o acesso geográfico é relevante para o desfecho do tratamento. A redução das taxas de morbidade e mortalidade depende da identificação precoce, pois, uma vez identificado o caso, o tratamento adequado e ágil concorre para reduzir os impactos da doença.

Neoplasmas de Mama; Análise Espacial; Acesso aos Serviços de Saúde; Sistemas de Informação

\section{Colaboradores}

E. X. G. Oliveira, E. C. P. Melo, R. S. Pinheiro, M. S. Carvalho e C. P. Noronha participaram da concepção da pesquisa, análise e processamento dos dados e redação do artigo.

\section{Agradecimentos}

À Fundação de Amparo à Pesquisa do Estado do Rio de Janeiro (FAPERJ). 


\section{Referências}

1. Garcia M, Jemal A, Ward EM, Center MM, Hao Y, Siegel RL, et al. Global cancer facts \& figures 2007. Atlanta: American Cancer Society; 2007.

2. Secretaria Nacional de Assistência à Saúde, Ministério da Saúde. Atlas de mortalidade por câncer no Brasil 1979-1999. Brasília: Instituto Nacional de Câncer; 2002.

3. Smith RA, Caleffi M, Albert US, Chen TH, Duffy SW, Franceschi D, et al. Breast cancer in limitedresource countries: early detection and access to care. Breast J 2006; 12 Suppl 1:S16-26.

4. Barry J, Breen N. The importance of place of residence in predicting late stage diagnosis of breast or cervical cancer. Health \& Place 2005; 11:15-29.

5. Knoke D, Kuklinski JH. Network analysis: basic concepts. In: Thompson G, Frances J, Levacic R, Mitchell J, editors. Markets, hierarchies and networks: the coordination of social life. London: Sage Publications; 1991. p. 173-82.

6. Bakis R. Les réseaux et les enjeux sociaux. Paris: Presses Universitaires de France; 1993.

7. Oliveira EXG, Travassos C, Carvalho MS. Acesso à internação hospitalar nos municípios brasileiros em 2000: territórios do Sistema Único de Saúde. Cad Saúde Pública 2004; 20 Suppl 2:S298-309.

8. Brasil. Portaria GM n ${ }^{\circ}$. 2.439, de 8 de dezembro de 2005. Institui a Política Nacional de Atenção Oncológica: Promoção, Prevenção, Diagnóstico, Tratamento, Reabilitação e Cuidados Paliativos, a ser implantada em todas as unidades federadas, respeitadas as competências das três esferas de gestão. Diário Oficial da União 2005; 19 dez.

9. Brasil. Portaria GM no. 3.535 , de 2 de setembro de 1998, republicada em 14 de outubro de 1998. Estabelece a estrutura dos centros de alta complexidade em Oncologia - CACON. Diário Oficial da União 1998; 14 out.

10. Nystuen JD, Dacey MF. A graph theory interpretation of nodal regions. Papers and Proceedings of the Regional Science Association 1961; 7:29-42.

11. Rabino GA, Occelli S. Understanding spatial structure from network data: theoretical considerations and applications. Cybergeo: European Journal of Geography, Systèmes, Modélisation, Géostatistiques 1997; 29. http://cybergeo.revues.org/index 2199.html (acessado em 10/Ago/2010).
12. Fundação Oswaldo Cruz. Sistemas de informações geográficas e análise espacial na saúde pública. Brasília: Ministério da Saúde; 2007. (Série B. Textos Básicos de Saúde) (Série Capacitação e Atualização em Geoprocessamento em Saúde, 2).

13. Fundação Oswaldo Cruz. Abordagens espaciais na saúde pública. Brasília: Ministério da Saúde; 2006. (Série B. Textos Básicos de Saúde) (Série Capacitação e Atualização em Geoprocessamento em Saúde, 1).

14. Brito C, Portela MC, Vasconcellos MTL. Sobrevida de mulheres tratadas por câncer de mama no estado do Rio de Janeiro. Rev Saúde Pública 2009; 43:481-9.

15. Schneider IJC, d'Orsi E. Sobrevida em cinco anos e fatores prognósticos em mulheres com câncer de mama em Santa Catarina, Brasil. Cad Saúde Pública $2009 ; 25: 1285-96$.

16. Huang B, Dignan M, Han D, Johnson O. Does distance matter? Distance to mammography facilities and stage at diagnosis of breast cancer in Kentucky. J Rural Health 2009; 25:366-71.

17. Penchansky R, Thomas JW. The concept of access: definition and relationship to consumer satisfaction. Med Care 1981; 19:127-40.

18. Haynes R, Pearce J, Barnett R. Cancer survival in New Zealand: ethnic, social and geographical inequalities. Soc Sci Med 2008; 67:928-37.

19. Tyczinsky JE, Hill TD, Berkel HJ. Why do postmenopausal Afro-American women do not benefit from overall breast cancer mortality decline? Ann Epidemiol 2006; 16:180-90.

20. Lagerlund M, Bellocco R, Karlsson P, Tejler G, Lambe M. Socio-economic factors and breast cancer survival: a population-based cohort study (Sweden). Cancer Causes Control 2005; 16:419-30.

21. Wang F, McLafferty S, Escamilla V, Luo L. Late-stage breast cancer diagnosis and health care access in Illinois. Prof Geogr 2008; 60:54-69.

Recebido em 01/Abr/2010

Versão final reapresentada em 13/Ago/2010 Aprovado em 13/Out/2010 